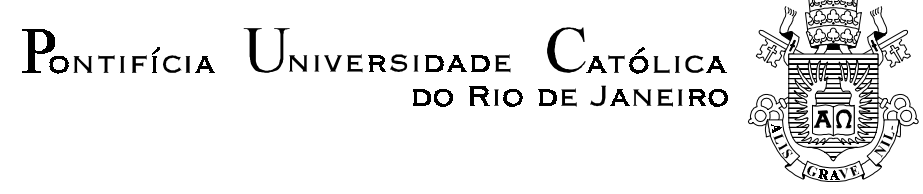

Regina Augusta Campos Sampaio

Espectro de Resposta de Projeto Uniformemente Provável para Sistemas Secundários Inelásticos

Tese de Doutorado

Tese apresentada como requisito parcial para obtenção do título de Doutor pelo Programa de PósGraduação em Engenharia Civil da PUC-Rio. Área de concentração: Estruturas.

Orientador: João Luis Pascal Roehl

Rio de Janeiro, outubro de 2003 


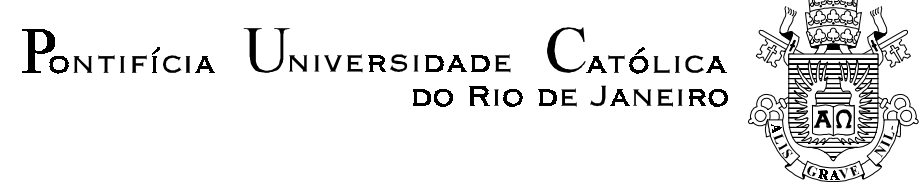

Regina Augusta Campos Sampaio

\title{
Espectro de Resposta de Projeto Uniformemente Provável para Sistemas Secundários Inelásticos
}

Tese apresentada como requisito parcial para obtenção do título de Doutor pelo Programa de Pós-Graduação em Engenharia Civil da PUC-Rio. Área de concentração: Estruturas. Aprovada pela Comissão Examinadora abaixo assinada.

\author{
João Luis Pascal Roehl \\ Orientador \\ PUC-Rio \\ Paulo Gonçalves Batista \\ PUC-Rio \\ Raul Rosas e Silva \\ PUC-Rio \\ José Eduardo Maneschy \\ ELETRONUCLEAR \\ Tereza Denyse Pereira de Araújo \\ UFC \\ Ney Augusto Dumont
}

Coordenador(a) Setorial do Centro Técnico Científico - PUC-Rio

Rio de Janeiro, 27 de outubro de 2003 
Todos os direitos reservados. É proibida a reprodução total ou parcial do trabalho sem autorização da universidade, da autora e do orientador.

\section{Regina Augusta Campos Sampaio}

Graduou-se em Engenharia Civil, pela Universidade Federal do Pará, em março de 1996. Ingressou no curso de mestrado em Engenharia Civil da PUC-Rio no ano de 1997, na área de concentração em Estruturas. Titulou-se Mestre em Ciências de Engenharia Civil: Estruturas pela PUC-Rio em março de 1999. Participou do projeto de colaboração entre a Eletronuclear e a PUC-Rio nos anos de 1998 a 2000.

Ficha Catalográfica

Sampaio, Regina Augusta Campos

Espectro de resposta de projeto uniformemente provável para sistemas secundários inelásticos / Regina Augusta Campos Sampaio ; orientador: João Luis Pascal Roehl. - Rio de Janeiro : PUC-Rio, Departamento de Engenharia Civil, 2003.

112 f. : il. ; $30 \mathrm{~cm}$

Tese (doutorado) - Pontifícia Universidade Católica do Rio de Janeiro, Departamento de Engenharia Civil.

Incluí referências bibliográficas.

1. Engenharia civil - Teses. 2. Espectro de resposta. 3.Inelasticidade. 4. Fator de dutilidade. 5. Sistemas secundários. I. Roehl, João Luis Pascal. II. Pontifícia Universidade Católica do Rio de Janeiro. Departamento de Engenharia Civil. III. Título. 


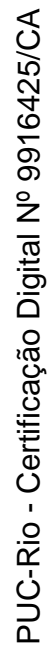

À Sophia 


\section{Agradecimentos}

\section{À Deus;}

Ao Prof. João Luis Pascal Roehl, amigo e mestre, pela compreensão das dificuldades da vida e pela dedicação em ensinar e transmitir sua sabedoria;

Ao CNPQ, à CAPES e à PUC-Rio pelo apoio financeiro;

Aos colegas da ELETRONUCLEAR sempre disponíveis a discutir e contribuir com a pesquisa;

Aos professores da pós graduação e funcionários do departamento de engenharia civil;

À Andreia e Denyse, obrigado pelas proveitosas discussões técnicas e mais ainda pela dedicada amizade;

À Ângela, Maria Fernanda e Paôla pelo carinho dedicado a mim e à minha filha nestes últimos anos;

A todos os amigos do mestrado e doutorado que dividiram comigo as alegrias e dificuldades destes anos de pós-graduação;

Aos meus pais, Carlos e Graça e aos meus irmãos, Antonio e João, por estarem sempre ao meu lado;

Às meninas Sophia e Fernanda, que renovam as esperanças nos nossos corações e;

Ao Giorgio, por dividir comigo a alegria de sermos uma família. 


\section{Resumo}

Sampaio, Regina Augusta Campos; Roehl, João Luis Pascal Espectro de resposta de projeto uniformemente provável para sistemas secundários inelásticos. Rio de Janeiro, 2003. 112p. Tese de Doutorado - Departamento de Engenharia Civil, Pontifícia Universidade Católica do Rio de Janeiro.

Estuda-se a consideração de comportamento inelástico do material na geração de espectros de resposta de projeto. Para tanto, trabalha-se sobre um sistema secundário simplificado acoplado a um pórtico de cinco andares com características dinâmicas ajustadas para modelar um sistema principal real, de uma usina nuclear. Faz-se um estudo paramétrico sobre estes sistemas acoplados onde são variados os parâmetros: fator de escoamento, a intensidade da excitação e o nível de amortecimento. É proposto um fator de dutilidade global formulado em termos de trabalho externo realizado sobre o sistema secundário. São obtidos espectros de dutilidade e de resposta. A análise de tais espectros fornece informações sobre o desempenho do sistema secundário e seus suportes e conclui por fatores de transposição entre os espectros elástico e inelástico. Propõe-se metodologia para obtenção de espectros de resposta elásticos e inelásticos que levam em conta o acoplamento entre os sistemas principal e secundário, o movimento relativo dos suportes e o compromisso probabilístico entre as ordenadas do espectro e a sismicidade da região expressa em termos de uma função densidade de espectro de potência objetivo para a aceleração do terreno. Um exemplo de obtenção de espectros de resposta acoplada de projeto uniformemente provável inelástico é apresentado.

\section{Palavras-chave}

sistemas secundários; comportamento inelástico; espectros de resposta; inelasticidade; fator de dutilidade 


\section{Abstract}

Sampaio, Regina Augusta Campos; Roehl, João Luis Pascal Uniformly probable project response spectra for inelastic secondary system. Rio de Janeiro, 2003. 112p. Dsc Thesis - Department of Civil Engineering, Pontifícia Universidade Católica do Rio de Janeiro.

The author's concern includes two main points in the subject of design response spectra generation for secondary systems in nuclear power plant structures: the consideration of inelastic behavior in the secondary systems materials and the production of uniformly probable design response spectra.

One works with a previously developed secondary system model attached to primary structure model tuned to the frequency range of a nuclear power plant building.

A global ductility factor is formulated relating the plastic to the overall work done by the seismic external forces on the secondary system. This factor together with a particular definition of the yielding factor allows one to determine elastic to inelastic spectrum transpose factors.

A methodology is proposed to generate uniformly probable coupled response spectra for multiply supported inelastic secondary systems.

The seismic excitation is prescribed by a target power spectrum density function of the ground acceleration and an internal pressure condition is added to the seismic action. Examples illustrate the application of this proposed methodology.

\section{Keywords}

secondary systems; inelastic behavior; response spectrum; inelasticity; ductility factor 


\section{Sumário}

1 Introdução

2 Situação no assunto 21

2.1. Aspectos Gerais $\quad 21$

2.2. Métodos de análise $\quad 21$

2.2.1. Definição da excitação sísmica 22

2.2.2. Domínios e métodos de análise 27

2.3. Análise modal espectral de sistemas secundários, suas razões e conseqüências. 30

2.3.1. Movimento relativo dos suportes. 32

2.3.2. Acoplamento dos sistemas 32

2.3.3. Espectros de resposta acoplada uniformemente provável. $\quad 37$

2.3.4. Consideração das não linearidades 38

3 Metodologia $\quad 45$

3.1. Estratégia 45

3.2. Sistema secundário simplificado 46

3.3. Sistema principal 50

3.4. Modelagem e análise no programa Ansys 52

3.4.1. Elementos PIPE20 e PIPE60 52

3.4.2. Comportamento não linear e inelástico na tubulação - elementos

PIPE20 e PIPE60 53

3.4.3. Elemento COMBIN40 e comportamento não linear dos suportes. 55

3.5. Carregamento no sistema secundário simplificado 56

3.6. Amortecimento 58

3.7. Fator de escoamento 60

3.8. Fator de dutilidade 60

3.9. Espectro de Resposta Acoplada de Projeto Uniformemente Provável Linear 
4 Ensaios e Resultados para o SSS acoplado ao SP 67

4.1. Programação dos ensaios $\quad 67$

4.2. Espectros de dutilidade 68

4.2.1. Apresentação 68

4.2.2. Análise geral para carga sísmica 68

4.2.3. Pressão interna $\quad 70$

4.2.4. Amortecimento $\quad 71$

4.2.5. Dutilidade dos apoios $\quad 79$

4.3. Espectros de resposta linear elástica 81

4.4. Espectros de resposta do sistema secundário simplificado 82

4.4.1. Apresentação 82

4.4.2. Análise Geral para a carga sísmica 83

4.4.3. Pressão interna 85

4.4.4. Amortecimento $\quad 85$

4.5. Avaliação do catraqueamento 92

4.6. Síntese das variações espectrais devidas à dutilidade 96

4.7. Exemplo de aplicação $\quad 97$

$\begin{array}{ll}\text { 4.7.1. Apresentação } & 97\end{array}$

4.7.2. Modelo do reator, excitação e sistema secundário. 97

4.7.3. Espectro de resposta acoplada uniformemente provável 101

$\begin{array}{lr}5 \text { Conclusões } & 105\end{array}$

6 Referências bibliográficas 108 


\section{Lista de figuras}

Figura 2.1 - Espectro de resposta. Acelerações espectrais são as amplitudes de aceleração máxima do $\mathrm{S} 1 \mathrm{GL}$ em resposta à mesma excitação. (Kramer, 1996)

Figura 2.2 - - Representação de um Espectro de quatro escalas logarítmicas.

Figura 2.3- Sistema com dois graus de liberdade (S2GL)

Figura 2.4- Primeiro e segundo modo do S2GL em função da freqüência do sistema secundário, normalizado pela freqüência do SP

Figura 2.5-Procedimento esquemático para obtenção de espectros de resposta acoplada em vários pontos (Valverde, 1998).

Figura 2.6 - Definição da faixa de deformação inelástica e da deformação por catraqueamento.

Figura 2.7 - a) Diagrama tensão deformação - baixa tensão média. b)

Diagrama tensão deformação - caso limite $\left(\varepsilon^{p c}=0\right)$.

Figura 2.8 - Catraqueamento transiente e assintótico- esquema

Figura 2.9 - Limites de comportamento do material para o caso de placa de comprimento unitário submetida a carga permanente de tração e carga cíclica de flexão.

Figura 3.1 - Representação esquemática do sistema secundário simplificado

Figura 3.2- Representação esquemática do sistema principal.

Figura 3.3 - Representação esquemática modelo de barras e placas do SP.

Figura 3.4 - Geometria do elemento PIPE20 52

Figura 3.5 - Geometria do elemento PIPE60 53

Figura 3.6 - Translação da superfície de escoamento com a progressão do escoamento. Encruamento cinemático. 55

Figura 3.7 - Elemento COMBIN40

Figura 3.8 - Sismo gerado artificialmente normalizado para uma aceleração máxima de $0,1 \mathrm{~g}$ e compatibilizado pelo espectro de resposta 
adotado para o trabalho.

Figura $3.9-$ Espectro de Fourier do sismo utilizado. $\ddot{v}_{g \max }=0,1 \mathrm{~g}$.

Figura 3.10 - Espectro de resposta de projeto em pseudo velocidade adotado para o trabalho, $\ddot{v}_{g \max }=0,1 \mathrm{~g}$.

Figura 3.11 - Densidade espectral de potência do sismo, $\ddot{v}_{g \max }=0,1 \mathrm{~g} . \quad 58$

Figura 3.12 - a) Força x tempo e deslocamento resultante. b) Relação bilinear histerética entre força e deslocamento.

Figura 3.13 - Procedimento esquemático de obtenção do Espectro de Resposta Uniformemente Provável. As ordenadas do espectro são obtidas a partir da fixação da reta horizontal que representa um nível de probabilidade $\mathrm{F}$ de não ser ultrapassada.

Figura 4.1 - Espectro de dutilidade para o SSS acoplado ao SP, parametrizado pelo fator de escoamento, C. Sismo1g e Sismo0,1g 72 Figura 4.2 - Espectro de dutilidade para o SSS acoplado ao SP, parametrizado pelo fator de escoamento, C. Sismo0,1g +Pi

Figura 4.3 - Espectro de dutilidade para o SSS acoplado ao SP, parametrizado pelo fator de escoamento, C. Sismo1g+Pi.

Figura 4.4 - Espectro de dutilidade para o SSS acoplado ao SP, parametrizado pelo fator de escoamento, C.Sismo1g+Pi-7\%.

Figura 4.5 - Espectro de dutilidade, $\mathrm{C}=0,2$. Comparação dos casos. $\quad 74$ Figura 4.6 - Função de transferência de acelerações do ponto de controle do sismo para os nós 206 e 210 do SSS acoplado ao SP. 75

Figura 4.7 - a) Representação esquemática do SSS com tubulação muito flexível. b) Representação esquemática do SSS com tubulação rígida. 75 Figura 4.8 - Períodos de regime linear elástico e plástico do SSS, $f_{0 s}=$ $0,25 \mathrm{~Hz}, \mathrm{C}=0,2$ 76

Figura 4.9 - Períodos de regime linear elástico e plástico do SSS, $f_{0 s}=5$ $\mathrm{Hz}, \mathrm{C}=0,2$ 76

Figura 4.10 - Períodos de regime linear elástico e plástico do SSS, $f_{0 s}=9$ $\mathrm{Hz}, \mathrm{C}=0,2$. 77

Figura 4.11 - Períodos de regime linear elástico e plástico do SSS, $f_{0 s}=$ $33 \mathrm{~Hz}, \mathrm{C}=0,2$. 
Figura 4.12 - Tensões equivalentes máximas para o elemento 399, C=1, $f_{0 s}=5 \mathrm{~Hz}$ e tensões de escoamento para $\mathrm{C}=0,2$.

Figura 4.13 - Tensões equivalentes máximas para o elemento $399, \mathrm{C}=1$, $f_{0}=9 \mathrm{~Hz}$ e tensões de escoamento para $\mathrm{C}=0,2$.

Figura 4.14 - Espectro de Fourier da resposta em deslocamento, u, do nó 85 do SP, com SSS acoplado, $f_{0}=5 \mathrm{~Hz}$ e C $=1$. Sismo1g.

Figura 4.15 - Espectro de resposta acoplada linear elástica - metodologia de Valverde (1998). Nó 46 do SP.

Figura 4.16 - Espectro de resposta acoplada linear elástica - metodologia de Valverde (1998). Nó 85 do SP.

Figura 4.17 - Espectro de resposta em pseudovelocidade do nó 206 do SSS. Sismo1g.

Figura 4.18 - Espectro de resposta em pseudovelocidade do nó 210 do SSS. Sismo1g.

Figura 4.19 - Espectro de resposta em pseudovelocidade para o nó 206 do SSS. Sismo1g+Pi.

Figura 4.20 - Espectro de resposta em pseudovelocidade para o nó 210 do SSS. Sismo1g+Pi.

Figura 4.21 - Espectro de resposta em pseudovelocidade do nó 206 do SSS. Sismo1g+Pi-7\%.

Figura 4.22 - Espectro de resposta em pseudovelocidade do nó 210 do SSS. Sismo1g+Pi-7\%.

Figura 4.23 - Espectro de resposta em pseudo velocidade normalizado pela resposta linear elástica $(\mathrm{C}=1)$ e parametrizado pelo fator de escoamento, C. Nó 206 do SSS. Sismo1g.

Figura 4.24 - Espectro de resposta em pseudo velocidade normalizado pela resposta linear elástica $(\mathrm{C}=1)$ e parametrizado pelo fator de escoamento, C. Nó 210 do SSS. Sismo1g.

Figura 4.25 - Espectro de resposta em pseudo velocidade normalizado pela resposta linear elástica $(\mathrm{C}=1)$ e parametrizado pelo fator de escoamento, C. Nó 206 do SSS. Sismo1g+Pi.

Figura 4.26 - Espectro de resposta em pseudo velocidade normalizado pela resposta linear elástica $(\mathrm{C}=1)$ e parametrizado pelo fator de 
escoamento, C. Nó 210 do SSS. Sismo1g+Pi.

Figura 4.27 - Espectros de resposta do nó 206 do SSS. $\mathrm{C}=0,2 . \xi=0 . \quad 91$

Figura 4.28 - Espectro de resposta em pseudo velocidade normalizado pela resposta linear elástica $(\mathrm{C}=1)$ e parametrizado pelo fator de escoamento, C. Nó 206 do SSS. Sismo1g+Pi-7\%.

Figura 4.29 - Espectro de resposta em pseudo velocidade normalizado pela resposta linear elástica $(\mathrm{C}=1)$ e parametrizado pelo fator de escoamento, C. Nó 210 do SSS. Sismo1 g+Pi-7\%.

Figura 4.30 - Relações entre tensão devido ao sismo e à pressão interna normalizadas pela tensão de escoamento. Elemento 399, seção i, ponto de integração $r$.

Figura 4.31 - Gráfico tensão-deformação. Elemento 399, seção i, ponto de integração $r$.

Figura 4.32 - Gráficos de tensão deformação para Sismo0,1g+Pi e Sismo0,1g. Elemento 399, seção i, ponto de integração r.

Figura $4.33-V / V_{0} \times f_{o s} / f_{o s p}$ em função de $\mathrm{C}, \boldsymbol{\xi}=0$ e $\xi=7 \%$

Figura 4.34 - Modelo da superestrutura do prédio do reator $\quad 100$

Figura 4.35 - Modelo da base do prédio do reator 100

Figura 4.36 - Densidade espectral de potência objetivo para o sítio de Angra 3 (Weston Geophisycal Research Inc,1972).

Figura 4.37 - Espectro de resposta acoplada de projeto uniformemente provável, elástico. Sistema secundário com $\xi=7 \%$. Sistema principal: modelo do prédio do reator da usina Angra 3. Acoplamento em um, dois e quatro pontos.

Figura 4.38 - Espectro de resposta acoplada uniformemente provável, elástico e inelástico. Sistema secundário $\operatorname{com} \xi=7 \%$. SP -modelo do prédio do reator da usina Angra 3. Acoplamento em um ponto. 


\section{Lista de tabelas}

Tabela 2.1 - Relação entre domínio de análise e regime do modelo

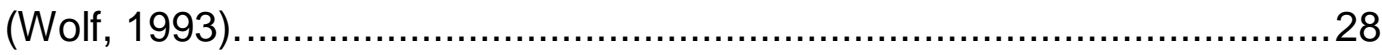

Tabela 3.1- Módulos de elasticidade e freqüências fundamentais do SSS 47

Tabela 3.2- Valores das relações, $f_{0 i} / f_{01}$, das 15 primeiras frequências do SSS para a sua frequência fundamental ................................................ 48

Tabela 3.3 - Valores das rigidezes inicial e final dos elementos de mola, $\mathrm{kN}$ ou $\mathrm{kN} \cdot \mathrm{m}$ 48

Tabela 3.4- Quinze primeiras freqüências naturais do sistema principal,

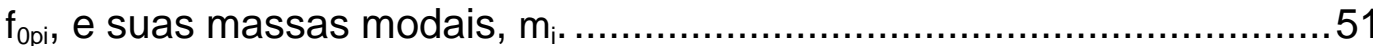

Tabela 3.5 - Comparação entre fatores de dutilidade................................62

Tabela 4.1 - Valores de $V / V_{0}$ para os casos não amortecidos. ............84

Tabela 4.2 - Fator de relação entre os espectros elástico e inelástico ....96 


\section{Lista de símbolos e abreviaturas}

\section{Romanos}

A Pseudo aceleração espectral

$\left|A_{n}\right| \quad$ Amplitude de Fourier

[C] Matriz de amortecimento

C Fator de escoamento

$\mathrm{C}_{\mathrm{ijkl}} \quad$ Matriz constitutiva

$\mathrm{c}_{\mathrm{i}} \quad$ Fator de participação no modo i

E Módulo de elasticidade

ERAUPE Espectro de resposta acoplada uniformemente provável elástico

ERAUPIN Espectro de resposta acoplada uniformemente provável inelástico

ERUP Espectro de resposta uniformemente provável

FAI $\quad$ Fator de amplificação instantâneo no modo i

$F_{j}(a) \quad$ Função distribuição de probabilidade da aceleração $a$ e freqüência $j$

$\mathrm{F}_{\mathrm{y}} \quad$ Força correspondente ao escoamento

$\mathrm{F}_{0} \quad$ Força máxima aplicada

$\mathrm{f}_{\mathrm{i}} \quad$ Freqüência i

$\mathrm{f}_{0 \mathrm{j}} \quad$ Freqüência natural $\mathrm{j}$ do sistema secundário

g Aceleração da gravidade

$H(\omega) \quad$ Função de transferência (função resposta a um harmônico unitário)

J Momento de inércia

[K] Matriz de rigidez

$\mathrm{k} \quad$ Coeficiente de rigidez, elemento da matriz de rigidez

$\mathrm{k}_{\mathrm{r}} \quad$ Coeficiente de rigidez rotacional

$\mathrm{k}_{\mathrm{t}} \quad$ Coeficiente de rigidez translacional

[M] Matriz de massa

m Massa, elemento da matriz de massa

$\mathrm{P}_{0} \quad$ Pressão externa no tubo

$\mathrm{P}_{\mathrm{i}} \quad$ Pressão interna no tubo 
$\mathrm{q}_{\mathrm{i}} \quad$ Relação entre as tensões provocadas pela pressão interna e a provocada pelo sismo

$\mathrm{S}_{\mathrm{ij}} \quad$ Tensor de tensões desviadoras

S1GL Sistema de um grau de liberdade

S2GL Sistema de dois graus de liberdade

SP Sistema principal

SS Sistema secundário

SSS Sistema secundário simplificado

T Período

$\mathrm{T}_{\mathrm{e}} \quad$ Trabalho elástico realizado pelas forças externas sobre o SSS

$\mathrm{T}_{\mathrm{m}} \quad$ Trabalho total realizado pelas forças externas sobre o SSS

$\mathrm{T}_{\mathrm{p}} \quad$ Trabalho plástico realizado pelas forças externas sobre o SSS

t Instante de tempo

$\mathrm{t}_{\mathrm{d}} \quad$ Duração da excitação

$\mathrm{t}_{1} \quad$ Duração do primeiro período de carregamento

U Deslocamento espectral

u,$v \quad$ Deslocamento

$\dot{u}, \dot{v} \quad$ Velocidade

$\ddot{u}, \ddot{v} \quad$ Aceleração

$u_{y}, v_{\mathrm{y}} \quad$ Deslocamento correspondente ao início do escoamento

$v_{\mathrm{g}} \quad$ Deslocamento do sismo

$v_{\mathrm{b}} \quad$ Deslocamento do nó de apoio do sistema secundário

$\ddot{v}_{g \max } \quad$ Aceleração máxima do sismo

V Pseudo velocidade espectral

$\mathrm{V}_{0} \quad$ Pseudo velocidade espectral - sistema secundário elástico

$v_{m} \quad$ Deslocamento máximo

$x_{r}(t) \quad$ Função amostra de um processo aleatório

$X_{n r} \quad$ Amplitude da série de Fourier 


\section{Gregos}

$\alpha_{\mathrm{ij}} \quad$ Variável de encruamento que determina o centro da superfície de escoamento

$\beta \quad$ Relação entre as freqüências do sistema secundário e a do principal

$\Delta \omega \quad$ Intervalo de freqüência circular

$\Delta \varepsilon^{\mathrm{p}} \quad$ Faixa de deformação plástica

$\delta \varepsilon^{\mathrm{p}} \quad$ Deformação plástica progressiva

$\varepsilon^{\mathrm{p}} \quad$ Deformação plástica

$\varepsilon^{\mathrm{pc}} \quad$ Deformação plástica à compressão

$\varepsilon^{\mathrm{pt}} \quad$ Deformação plástica à tração

$\phi_{\mathrm{i}} \quad$ Modo de vibração com frequiência $\omega_{0 i}$

$\Phi_{r r} \quad$ Densidade espectral de potência da resposta $\mathrm{r}$

$\Phi_{\ddot{v} g \text { v̈g }}^{0} \quad$ Densidade espectral de potência objetivo da aceleração do sismo

Relação entre as massas do sistema secundário e a do principal

$\eta \quad$ Relação entre as freqüências do sistema acoplado e a do principal

$\lambda_{\mathrm{i}} \quad$ Momento espectral de iésima ordem

$\mu, \mu^{\prime}, \mu^{*} \quad$ Fator de dutilidade

$\rho$

Relação entre os coeficientes de rigidez final e inicial do gráfico força deslocamento bilinear

$\sigma \quad$ Tensão

$\sigma_{0} \quad$ Tensão equivalente elástica máxima

$\sigma_{\mathrm{b}} \quad$ Tensão devido a flexão

$\sigma_{\text {eq }} \quad$ Tensão equivalente que depende do critério de escoamento

$\sigma_{\mathrm{p}} \quad$ Tensão devido a carregamento primário

$\sigma_{\mathrm{y}} \quad$ Tensão de escoamento

$\omega \quad$ Freqüência circular

$\omega_{0 \mathrm{i}} \quad$ Freqüência circular natural i do SS

$\omega_{0 \text { pi }} \quad$ Freqüência circular natural i do sistema principal

$\xi \quad$ Fator de amortecimento 